\title{
ON THE FROBENIUS ENDOMORPHISMS OF FERMAT AND ARTIN-SCHREIER CURVES
}

\author{
ROBERT F. COLEMAN
}

(Communicated by Larry J. Goldstein)

\begin{abstract}
The following article offers an explanation of the relationship of Jacobi and Gauss sums to Fermat and Artin-Schreier curves which is an analogue of the proof of Stickleberger's theorem.
\end{abstract}

1. Correspondences. The connection between cubic Fermat curves and cubic Jacobi sums was first observed by Gauss $[\mathbf{G}]$, who used it to study such sums. That one can compute the number of points on a Fermat curve over a finite field using Jacobi sums has long been known. The same is true for Artin-Schreier curves and Gauss sums and were applied by Davenport and Hasse [D-H] to compute Artin $L$-functions associated with such curves. This computation, in turn, yielded the Hasse-Davenport identity. Ultimately, Weil $[\mathbf{W}]$ interpreted these computations via a Lifshetz fixed point formula by showing that the eigenvalues of Frobenius acting on what is now known as the étale cohomology groups of Fermat curves are Jacobi sums and on Artin-Schreier curves are Gauss sums. This can be interpreted as an identity which says, in a suitable sense, that Frobenius is a Gauss or Jacobi sum (see below). One can now use this to produce annihilators of the divisor class groups of these curves which are also given by the Brumer-Stark conjecture for function fields proven in $[\mathbf{T}]$. Below, we will give an elementary proof of the aforementioned identity which is analogous to Stickelberger's proof of Stickelberger's theorem. We just write down especially simple functions with appropriate divisors.

Let $p$ be a rational prime and $m$ an integer prime to $p$. Let $K$ be a field of characteristic $p$. Let $A_{m}$ and $F_{m}$ denote the complete nonsingular curves over $K$ with affine equations:

$$
A_{m}: y^{p}-y=x^{m}, \quad F_{m}: u^{m}+v^{m}=1 .
$$

Suppose $q=p^{f} \equiv 1 \bmod m$ and suppose $K \supseteq \mathbb{F}_{q}$. Define the homomorphisms

$$
\psi: \mathbb{F}_{q} \rightarrow \operatorname{Aut}\left(A_{m}\right), \quad \chi: F_{q} \rightarrow \operatorname{Aut}\left(A_{m}\right), \quad \chi_{0}, \chi_{1}: F_{q} \rightarrow \operatorname{Aut}\left(F_{m}\right)
$$

by

$$
\begin{array}{cc}
\psi(a)(x, y)=\left(x, y+T_{\mathbf{F}_{q} / \mathbf{F}_{p}}(a)\right), & \chi(b)(x, y)=\left(b^{(q-1) / m} x, y\right), \\
\chi_{0}(b)(u, v)=\left(b^{(q-1) / m} u, v\right), & \chi_{1}(b)(u, v)=\left(u, b^{(q-1) / m} v\right)
\end{array}
$$

for $a \in \mathbf{F}_{q}^{+}$and $b \in \mathbf{F}_{q}^{*}$.

Now recall that a correspondence between a curve $X$ to a curve $Y$ is a divisor $Z$ on $X \times Y$ with no vertical or horizontal components. Among other things, $Z$ gives

Received by the editors April 15, 1986 and, in revised form, December 9, 1986.

1980 Mathematics Subject Classification (1985 Revision). Primary 11 G20. 
a way of producing a divisor on $Y$ from a divisor on $X$ by the formula:

$$
Z(D)=\pi_{2}\left(\pi_{1}^{-1}(D) \cdot Z\right)
$$

where $\pi_{1}$ and $\pi_{2}$ are the first and second projections and "." is the intersection product. In fact, this induces a homomorphism from the divisor class group of $X$ over extensions of $K$ to that of $Y$. We say two correspondences are equivalent if they induce the same such homomorphism for all such extensions. We regard a nonconstant map from $X$ to $Y$ as the correspondence given by its graph. Finally, one can add two correspondences and if $X=Y$, one can compose two correspondences. (For more details on correspondences see [W].)

Now set

$$
G_{q}(m)=-\sum_{b \in \mathbf{F}_{q}} \chi(b) \psi(b), \quad J_{q}(m)=-\sum_{b \in \mathbf{F}_{q}^{*}-\{1\}} \chi_{0}(b) \chi_{1}(1-b),
$$

regarded as correspondences on the corresponding curve. For any curve over $\mathbb{F}_{q}, \phi_{q}$ will denote the Frobenius endomorphism (which raises the coordinates of a point to the $q$ th power).

THEOREM A. Under equivalence of correspondences, we have $\phi_{q}=G_{q}(m)$ on $A_{m}$ and $\phi_{q}=J_{q}(m)$ on $F_{m}$.

ProOF. We may suppose $K$ is algebraically closed. Let $\infty$ denote the point at infinity on $A_{m}$. For $P$, a finite point on $A_{q-1}$ over $K$ such that $x(P) \neq 0$, let

$$
g_{P}=(y-y(P))-\sum_{i=0}^{f-1}(x / x(P))^{p^{i}}
$$

in $K\left(A_{q-1}\right)$. We claim that, if $x(P)^{q-1} \notin \mathrm{F}_{q}$,

$$
\left(g_{P}\right)=\left(\phi_{q}-G_{q}(q-1)\right)(P-\infty) \text {. }
$$

Indeed, first observe that $g_{P}$ has pole of order $p^{f}$ at $\infty$ and no other poles. Next, it vanishes at the $(q-1)$ points

$$
\chi(b) \psi(b) P=\left(b x(P), y(P)+T_{\mathbf{F}_{q} / \mathbf{F}_{p}}(b)\right),
$$

$b \in \mathbf{F}_{q}^{*}$. Finally, it vanishes at $\phi_{q}(P)=\left(x(P)^{q}, y(P)^{q}\right)$, which is distinct from the previous points if $x(P)^{q-1} \notin \mathrm{F}_{q}$. This proves the claim and establishes the theorem for $A_{q-1}$ since it suffices to check that $\left(\phi_{q}-G_{q}(q-1)\right)(P-\infty)$ is principal for all but finitely many $P$. (Note, however, that $x(P)^{p^{f-1}} g_{P}$ extends to a function which works for all $P$.)

Let $\infty$ denote any point at infinity on $F_{m}$. If $Q$ is a finite point on $F_{q-1}$ over $K$ such that $u(Q) \neq 0$ and $v(Q) \neq 0$, set

$$
j_{Q}=1-(u / u(Q)+v / v(Q)) .
$$

Then, by an argument similar to the above,

$$
\left(j_{Q}\right)=\left(\phi_{q}-J_{q}(q-1)\right)(Q-\infty)
$$

if $\left(u(Q)^{(q-1)}, v(Q)^{(q-1)} \notin \mathrm{F}_{q}\right)$. This completes the proof for $m=q-1$. (Note, similarly to the above, $u(P) v(P) j_{P}$ extends to a function which works for all $P$.) 
Now let $f: A_{q-1} \rightarrow A_{m}$ be the map

$$
(x, y) \mapsto\left(x^{(q-1) / m}, y\right) .
$$

Let $N: K\left(A_{q-1}\right) \rightarrow K\left(A_{m}\right)$ denote the corresponding norm map on function fields. Then

$$
\left(N g_{P}\right)=\left(\phi_{q}-G_{q}(m)\right)(f(P)-\infty),
$$

which completes the proof for $A_{m}$. A similar argument completes it for $F_{m}$.

COROLlaRY. Suppose $K=\mathrm{F}_{q}$. Then $1-G_{q}(m)$ annihilates the divisor class group of $A_{m}$ and $1-J_{q}(m)$ annihilates the divisor class group of $F_{m}$.

II. Relationship to Brumer-Stark. For simplicity, we will only discuss the case of Fermat curves in this section which we consider as curves over $F_{q}$. We will employ the notation of [T, Chapter V]. Let $G_{m}$ denote the group of automorphisms of $F_{m}$ generated by the images of $\chi_{0}$ and $\chi_{1}$. Let $l$ be a prime different from $p$. Let $H$ denote the first $l$-adic étale homology group of $F_{m}$. Then it is easy to see, and well known, that

$$
H \otimes \mathbf{z}_{l} \mathbb{Q}_{l}\left(\mu_{m}\right)
$$

breaks up into one-dimensional eigenspaces for the action of $G_{m}$. In fact, if $\psi$ is a character of $G_{m}$, then

(*) $\operatorname{dim}_{\mathbb{Q}_{l}\left(\mu_{m}\right)}\left(H^{(\psi)} \otimes \mathbb{Q}_{l}\left(\mu_{m}\right)\right)= \begin{cases}0 & \text { if } \psi \circ \chi_{0}=1, \text { or } \psi \circ \chi_{0} \chi_{1}^{-1}=1, \\ 1 & \text { otherwise. }\end{cases}$

The map $(u, v) \rightarrow u$ makes $F_{m}$ an Abelian cover of $\mathbf{P}^{1}$ with Galois group $G$. For a closed point $x$ of $\mathbf{P}^{1}$, we let $I_{x}$ denote the inertia subgroup of $F_{m}$ corresponding to $x$. We also define the element of $\mathbb{Q}\left[G_{m}\right]$,

$$
F_{x}=\left(1 /\left|I_{x}\right|\right) \sum \sigma_{v}^{-1} I_{v}
$$

where $\sigma_{v}^{-1}$ denotes any Frobenius element above $x$. In particular, $F_{i}=(1 / m) \sum I_{i}$ if $i \in\{0,1, \infty\}$. We also let $\varepsilon=\left(1 / \mathrm{m}^{2}\right) \sum G$. Because $I_{0}, I_{1}$ or $I_{\infty}$ equals the kernel of $\chi_{0}, \chi_{1}$ or $\chi_{0} \chi_{1}^{-1}$ respectively it follows from $(*)$ that as a $\mathbb{Q}\left[G_{m}\right]$ module

$$
H \approx \alpha \mathbb{Q}\left[G_{m}\right]
$$

where $\alpha=1+2 \varepsilon-\left(F_{0}+F_{1}+F_{\infty}\right)$.

As in $[\mathbf{T}]$, we set

$$
\Theta(T)=\prod\left(1-F_{v} T^{\operatorname{deg} v}\right)^{-1} \in \mathbb{Q}\left[G_{m}\right][[T]] .
$$

Using (**), Theorem A and Theorem V.2.5 of [T] we easily deduce

THEOREM B.

$$
\Theta(T)=\left(1-\alpha J_{q}(m) T\right) /(1-\varepsilon T)(1-\varepsilon q T),
$$

where here we regard $J_{q}(m)$ as an element of $\mathbb{Q}\left[G_{m}\right]$.

Let $S=\{0,1, \infty\}$, and

$$
\Theta_{S}(T)=\Theta(T)\left(1-F_{0} T\right)\left(1-F_{1} T\right)\left(1-F_{\infty} T\right) .
$$

Then according to Theorem V.1.2 of $[\mathbf{T}],(q-1) \Theta(1) \in \mathbb{Z}\left[G_{m}\right]$ and if $D$ is a divisor of degree zero on $F_{m}$, then there exists a function $f$ on $F_{m}$ such that 
$(f)=(q-1) \Theta(1) D$ and such that the field $\mathbb{F}_{q}\left(F_{m}\right)\left(f^{1 /(q-1)}\right)$ is Abelian over $\mathbb{F}_{q}(u)$. We can see this explicitly using the results in $\S I$.

Set $J=J_{q}(m)$. First of all, an easy calculation shows

$$
\Theta_{S}(t)=(1-\alpha J)\left(1+\left(\varepsilon-\left(F_{0}+F_{1}+F_{\infty}\right)\right) T+\varepsilon T^{2}\right) /(1-\varepsilon q T)
$$

and

$$
\Theta_{S}(1)=(1-\alpha J) \alpha=(1-J) \alpha
$$

since $\alpha$ is an idempotent. Moreover,

$$
(1-J) \alpha=(1-J)+(2(q-1)-3 m) \varepsilon
$$

since it is easy to check that $(1-J) F_{i}=m \varepsilon, i \in S$. In particular, $(q-1)(1-J) \alpha \in$ $\mathbb{Z}\left[G_{m}\right]$, since $m$ divides $q-1$.

Now let $D$ be a divisor of degree 0 on $F_{m}$. Then we know from $\S$ I that $(1-J) D$ is principal. Moreover, $m^{2} \varepsilon D$ is just the "norm" of $D$ to $\mathbf{P}^{1}$, so is the divisor of a function on $\mathbf{P}^{1}$. It follows immediately that $(q-1) \Theta_{S}(1) D$ is the divisor of a function on $F_{m}$, which is an $m$ th power times a rational function in $u$. Hence its $m$ th root generates an Abelian extension of $F_{q}(u)$ as predicted. In fact, using the results of $\S \mathrm{I}$, one can write this function down explicitly (although it is somewhat complicated). (Note, in general, such explicit functions have been exhibited by Hayes using Drinfeld modules [H].)

\section{REFERENCES}

[G] K. F. Gauss, Disquisitiones arithmeticae, Werke, Vol. I, 1901.

[D-H] H. Davenport and H. Hasse, Die Nullstellen der Kongruenzzetafunktionen in gewessin zyklischen Fällen, Crelles J. 172 (1935), 151.

[H] D. Hayes, Stickelberger elements in function fields, Compositio Math. 55 (1985), 209-239.

[T] J. Tate, Les conjectures de Stark sur les fonctions L d'Artin en $s=0$, Birkhäuser, Boston, Mass., 1984.

[W] A. Weil, Courbes algébriques et variétés abéliennes, Hermann, Paris, 1971.

Department of MAthematics, UNiversity of CALIFornia, Berkeley, CALiforNIA 94720 